\title{
Frecuencia de Cardiopatías Congénitas en fetos con Síndrome Genético, en el Hospital Regional de Concepción, Chile
}

\author{
DOI: $10.46981 /$ sfjhv2n2-003
}

Received in: January 1st, 2020

Accepted in: March 30th, 2020

\author{
Alejandro Alvarez Jara \\ Médico-Cirujano \\ Pediatra y Cardiólogo Infantil. \\ Unidad de Exploración Fetal, Hospital Guillermo Gran Benavente Concepción. \\ Colaborador docente del Departamento de Obstetricia Facultad de Medicina Universidad de \\ Concepción, Chile. \\ Dirección: O’Higgins 1320 Concepción, Chile. \\ E-mail: alalvarez1964@icloud.com
}

\section{RESUMEN}

Introducción: Las cardiopatías congénitas son las anomalías congénitas severas más frecuentes. Existe una mayor prevalencia de defectos cardiacos asociados a síndromes genéticos. Objetivo: mostrar nuestra experiencia en el diagnóstico antenatal de cardiopatías congénitas en fetos de embarazadas con cariograma patológico. Materiales y métodos: estudio retrospectivo descriptivo de todas las embarazadas a las que se realizó estudio genético por amniocentesis por sospecha de genopatía entre los años 2012 a 2016 en la unidad de exploración fetal del Hospital Regional de Concepción, Chile. ,Resultados: se efectuó estudio genético a 80 embarazadas. La edad promedio de las madres era de 30 años (17-44), con una edad gestacional promedio 29 semanas (22-35). En 45 el examen fue normal. En 25 se encontró alguna alteración cromosómica. No hubo desarrollo celular en 10, en estas el examen genético fue inconcluso. De las 25 que tuvieron estudio genético positivo, el resultado del ecocardiograma fetal, pesquiso canal atrioventricular común en 8(32\%), comunicación interventricular en 3(12\%), cardiopatía congénita compleja en 5(20\%), en 4(16\%) el ecocardiograma fetal fue normal y en $5(20 \%)$ no fue factible efectuar el examen. Conclusiones: La cardiopatía congénita más frecuente fue el canal atrioventricular, seguida de la comunicación interventricular. Todos los fetos con trisomía 13 presentaron defectos cardiacos cianóticos complejos.

Palabras-Claves: defectos congénitos cardiacos, genopatía, ecocardiograma fetal.

\section{INTRODUCCIÓN}

El 3 a $4 \%$ de todos los recién nacidos presentan una malformación congénita importante al nacer, siendo las cardiopatías congénitas las más frecuentes con una incidencia de 8x 1000 nacidos vivos. Y constituyen una de las primeras causas de mortalidad infantil. Las cardiopatías congénitas son las anomalías congénitas severas más frecuentes ${ }^{1}$. Existe una mayor prevalencia de defectos cardiacos asociados a síndromes genéticos en algunas condiciones con incidencia tan alta como en el 90\% de los $\operatorname{casos}^{2}$. Las alteraciones genéticas más frecuentes son las anomalías numéricas por ganancia o pérdida de cromosomas con una alta incidencia de aborto esponateno ${ }^{3}$. 
Mostramos en este trabajo una serie de fetos de embarazos con cariograma patológico y su asociación con defecto congénitos cardiacos, cuyo diagnóstico se realizó con ecocardiograma fetal.

Objetivo: mostrar nuestra experiencia en el diagnóstico antenatal de cardiopatías congénitas en fetos de embarazadas con cariograma patológico.

\section{MATERIALES Y MÉTODOS:}

Estudio retrospectivo descriptivo de todas las embarazadas a las que se realizó estudio genético por amniocentesis por sospecha de genopatía y a las cuales se les solicito un ecocardiograma que fue realizado por especialista en cardiología pediátrica y fetal entre los años 2012 a 2016 en la Unidad de Exploración Fetal del Hospital Regional de Concepción, Chile.

\section{RESULTADOS:}

Se efectuó estudio genético a 80 embarazadas durante el periodo estudiado. La edad promedio de las madres era de 30 años con un rango 17-44 años, tenían una edad gestacional promedio 29 semanas al momento de su evaluación con un rango de edad gestacional de 22-35 semanas. En 45 fetos el cariograma fue normal. El cariograma presentó una positividad del 31\% de los casos estudiados. En 25 se encontró alguna alteración cromosómica en el cariograma hubo 12 fetos con trisomía 18, 7 con trisomía 21, 3 con trisomía 13, tetraploidea un caso, un feto con síndrome de Turner y otro feto con 13q. No hubo desarrollo celular en 10 muestras de líquido amniótico, en estas el examen genético fue inconcluso. El ecocardiograma fetal fue realizado por un solo operador y mostro en los pacientes con cariograma alterado las siguientes patologías tabla $\mathrm{n}^{\mathbf{0}} 1$.

\section{FRECUENCIA DE DEFECTOS CARDIACOS CONGÉNITOS EN FETOS CON ANOMALÍAS CROMOSÓMICAS.}

\begin{tabular}{|c|c|c|c|c|c|c|c|}
\hline $\begin{array}{l}\text { Defecto } \\
\text { Cardiaco }\end{array}$ & Trisomía 21 & Trisomía 18 & Trisomía 13 & Tetraploidea & Síndrome Turner & 13q- & Total \\
\hline CAVC & 3 & 5 & & & & & $8(32 \%)$ \\
\hline CIV & 1 & 1 & & 1 & & & $3(12 \%)$ \\
\hline CAVC+TGA & & 1 & & & & & $1(4 \%)$ \\
\hline AM+DSVD & & & 1 & & & & $1(4 \%)$ \\
\hline TA tipo I & & & 1 & & & & $1(4 \%)$ \\
\hline TF & & & 1 & & & & $1(4 \%)$ \\
\hline SHVI & & 1 & & & & & $1(4 \%)$ \\
\hline Normal & 3 & 1 & & & & & $4(16 \%)$ \\
\hline S/E & & 3 & & & 1 & 1 & $5(20 \%)$ \\
\hline Total & 7 & 12 & 3 & 1 & 1 & 1 & $25(100 \%)$ \\
\hline
\end{tabular}

CAVC: canal atrio ventricular común, CIV: comunicación interventricular, TGA: transposición de los grandes vasos. AM: atresia mitral, DSVD: doble salida del ventrículo derecho, TA: truncus arterioso, TF: tetralogía de Fallot, SHVI: síndrome de hipoplasia del ventrículo izquierdo, S/E: sin ecocardiograma fetal. 


\section{DISCUSIÓN:}

El estudio prenatal de anomalías cromosómicas debe ser parte del control prenatal en casos de fetos con riesgo alto y sospecha de genopatía. La amniocentesis para estudio genético es un examen no exento de riesgo, pero realizado en un centro especializado es de gran utilidad para el manejo y conducta a seguir con las pacientes. En países donde la interrupción del embarazo es una opción, se intenta hacer el diagnóstico de malformación cardiaca fetal en el primer trimestre del embarazo. Nosotros encontramos una positividad del cariograma por amniocentesis similar a la de otras series de la literatura medica ${ }^{6}$.

En nuestra serie estudiada pesquisamos un mayor porcentaje de síndrome de Edward (trisomía 18) siendo esta genopatía la con más alta asociación a cardiopatía congenitas ${ }^{2,6}$. La genopatía más frecuente descrita en la literatura con una incidencia de 1 por 680 nacidos vivos es síndrome de Down (trisomía 21) y estos pacientes presentan entre 40-50\% defectos cardiacos congénitos ${ }^{4,5,7}$. Los niños con síndrome de Down presentan una frecuencia de cardiopatías congénitas más alta que la población general. Según las series, presentan algún tipo de anomalía que requiere el control por el cardiólogo pediatra y en algunos casos cirugía correctora con una buena sobrevida ${ }^{8}$. El síndrome de Patau (trisomía 13) se asocia con alta frecuencia con defectos cardiacos cianóticos complejos. Este último y el síndrome de Edward siendo ambas anomalías letales ${ }^{3,5}$. La cardiopatía congénita más frecuente encontrada fue el canal atrio ventricular común asociado a la trisomía 18 y 21, seguida de la comunicación interventricular esto difiere de otras publicaciones donde el defecto más frecuentemente encontrado es la comunicación interventricular ${ }^{9.10}$. Todos los fetos con trisomía 13 presentaron defectos cardiacos complejos de tipo cianótico, cardiopatías cono-troncales esto es similar a lo descrito en la literatura ${ }^{11}$. Hoy en día las madres inician sus gestaciones a mayor edad y no debemos olvidar, que las tasas de genopatías aumentan exponencialmente con el avance de la edad materna especialmente para las trisomías 21,18 y $13^{12}$.

\section{CONCLUSIÓN:}

Creemos que es de suma importancia realizar estudio genético y ecocardiograma fetal en las embarazadas de alto riesgo de genopatía, para poder efectuar un consejo médico adecuado y derivar in útero al centro cardiovascular para su manejo a los fetos que corresponda según su patología y así poder mejor la morbimortalidad en los casos de enfermedades no letales. 


\section{BIBLIOGRAFÍA}

1. Mayorga H Carla, Rodríguez A Juan Guillermo, Enríquez G Gabriela, Alarcón R Jaime, Gamboa W Constanza, Capella S Daniela et al. Cardiopatías congénitas: diagnóstico prenatal y seguimiento. Rev. Chil. Obstet. Ginecol. 2013 Oct; 78(5): 349-356.

2. Hagit Shani, Pe'er dar, and Mark I. Evans. Genetic and cardiac anomalies. Fetal Cardiology Third Edition 2019 CRC Press Taylor \& Francis Group. Cap 49 Pag: 643-650.

3. Mora AP, Paredes D, Rodríguez O, Quispe E, Chavesta F, Klein de Zihelboim E, De Michelena M. Anomalías cromosómicas en abortos espontáneos. Rev Per Ginecol Obstet. 2016;62(2):141-51.

4. Ritu Mogra and Jon Hyett. Cardiac defect in chromosomally abnormal fetuses. Fetal Cardiology Third Edition 2019 CRC Press Taylor \& Francis Group. Cap 50 Pag: 651-664.

5. Aracena A. Mariana. Cardiopatías congénitas y síndromes malformativos-genéticos. Rev. Chil. Pediatr. 2003 Jul; 74 (4): 426-431.

6. Huamán G Moisés, Quiroga de Michelena María Isabel, St. Martin Brad, Huamán Moisés. Diagnóstico prenatal de anomalías cromosómicas: Chorionic villous sampling and amniocentesis for fetal karyotyping Biopsia de vellosidades coriales y amniocentesis para cariotipo fetal. Rev. Peru. Ginecol. Obstet. 2016 Jul; 62 (3): 269-277.

7. Miguel A. Ruz-Montes, Eliana M. Cañas-Arenas, María A. Lugo-Posada, María A. Mejía-Carmona, Manuela Zapata-Arismendy, Laura Ortiz-Suárez y María I. Henao-Montaño. Cardiopatías congénitas más frecuentes en niños con síndrome de Down. Rev. Colomb. Cardiol. 2017; 24(1):66-70.

8. Núñez Gómez F, López-Prats, Lucea JL. Cardiopatías congénitas en niños con síndrome de Down.

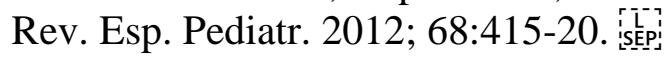

9. Clur SA, Van Brussel PM, Mathijssen IB, Pajkrt E, Ottenkamp J, Bilardo CM. Audit of 10 years of referrals for fetal echocardiography. Prenat Diagn 2011; 31:1134-40.

10. Aguilera S, Rodríguez J, Enríquez G, et al. Cardiopatías congénitas con diagnóstico prenatal: seguimiento. Rev Chil Obstet Ginecol 2006; 71:320-6.

11. Ferrer Q, Albert D, Arevalo S, Soro G, et al. Prenatal diagnosis and treatment planning of conotruncal anomalies: fetal echocardiographic features, associated anomalies and outcome. Ultrasound

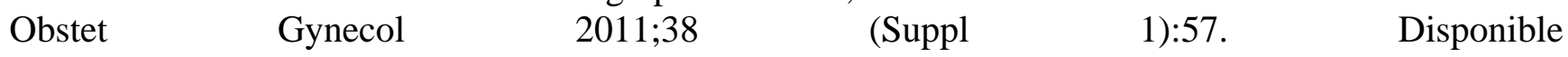
en: http://onlinelibrary.wiley.com/doi/10.1002/uog.9263/pdf

12. Ferguson-SmithMA, Yates JRW. Maternalage-speci crates for chromosomes aberrations and factors in uencing them: report of a collaborative European study on 52965 amniocenteses. Prenal Diagn. 1984 Spring;4 Spec N.5:44. http://onlinelibrary.wiley.com/doi/10.1002/pd.1970040704/full. 